\title{
Computerized Cognitive Training in Children With Autism and Intellectual Disabilities: Feasibility and Satisfaction Study
}

Songpoom Benyakorn ${ }^{1,2,3}$, MD; Catrina A Calub ${ }^{2,3}$, MA; Steven J Riley ${ }^{2,3}$, MS; Andrea Schneider ${ }^{3,4}$, PhD; Ana-Maria Iosif $^{5}, \mathrm{PhD}$; Marjorie Solomon ${ }^{2,3}, \mathrm{PhD}$; David Hessl ${ }^{2,3}, \mathrm{PhD}$; Julie B Schweitzer ${ }^{2,3}, \mathrm{PhD}$

\footnotetext{
${ }^{1}$ Department of Psychiatry, Faculty of Medicine, Srinakharinwirot University, Ongkharak, Nakhonnayok, Thailand

${ }^{2}$ Department of Psychiatry and Behavioral Sciences, University of California, Davis, Sacramento, CA, United States

${ }^{3}$ MIND Institute, University of California, Davis, Sacramento, CA, United States

${ }^{4}$ Department of Pediatrics, University of California, Davis, Sacramento, CA, United States

${ }^{5}$ Department of Public Health Sciences, University of California, Davis, Davis, CA, United States
}

\section{Corresponding Author:}

Julie B Schweitzer, $\mathrm{PhD}$

Department of Psychiatry and Behavioral Sciences

University of California, Davis

2825 50th St, MIND Institute

Sacramento, CA, 95817

United States

Phone: 19167030294

Email: jschweitzer@ucdavis.edu

\section{Abstract}

Background: Researchers are increasingly interested in testing and developing computerized cognitive training interventions for individuals with autism spectrum disorder due to the limited accessibility of treatments for this disorder. Understanding the feasibility of testing cognitive interventions for this population is critical, especially for individuals with ASD who have low to moderate intellectual ability.

Objective: The aim of the study was to evaluate the feasibility of computerized cognitive training as measured by attrition rate and a parent satisfaction survey.

Methods: A total of 26 participants aged 8-17 years with an autism spectrum disorder diagnosis and significant intellectual impairment were enrolled (mean age 11.1 years). They were instructed to complete 25 sessions of Cogmed Working Memory Training in 5 to 6 weeks with coach assistance. Attrition rate and parent satisfaction surveys were measured after the completion of training.

Results: Most participants (96\%, 25/26) completed the training and indicated high satisfaction (>88\%). However, among the participants who completed the training, 5 participants (19\%) were unable to finish in 6 weeks, the recommended training period by Cogmed. Parents noted various positive (eg, voice-overs) and negative (eg, particular graphic and sounds associated with a stimulus) features of the game that they thought affected their child's response.

Conclusions: Children with autism spectrum disorder and intellectual impairments can successfully participate in computerized cognitive training interventions but may require additional weeks to complete the training beyond the time needed for children without intellectual impairments. The overall completion rate, with extended time to complete the training, was high. Developers of cognitive training programs for this population should take into account potential issues regarding the noise level of stimuli and characteristics of the visual graphics.

(JMIR Ment Health 2018;5(2):e40) doi: 10.2196/mental.9564

\section{KEYWORDS}

autism; training; working memory; intellectual disability; treatment adherence; satisfaction 


\section{Introduction}

\section{Developing Novel Interventions for Treating Autism}

Autistic spectrum disorder (ASD) is a common neurodevelopmental disorder, with an overall prevalence of $1.47 \%$ of children in the United States [1]. Traditionally, interventions for ASD have relied on models of individual training with a therapist or small group therapy with a therapist or caregiver [2]. There are inherent limitations in this model, however, due to the limited access to trained professionals and a relatively high cost of service delivery. With concerns about the rising prevalence of ASD [1], there is an urgent need for additional and supplemental interventions to address deficits associated with ASD. Increasingly, interventions using computer technology are being considered to fill this gap [3,4]. A critical step before implementing a technological intervention for persons with ASD is assessing whether its use is feasible and acceptable to caregivers. Moving new potential interventions rapidly into the community requires partnerships with the stakeholders, in which they can give feedback on their experience [5]. As noted by Kelley and colleagues [5], ongoing input from the perspective of the stakeholders (in this case, parents) is key throughout the translational phase as a potential intervention moves from inception, discovery to delivery. Input and feedback from stakeholders can then be used to develop new interventions and refine those in the pipeline. The funding agency for this project, the Department of Defense (Autism Research Program), through a Pilot Award, also recognized the need to first test the feasibility of a potential therapy before it would be tested in a full-scale, randomized control trial. Findings from feasibility studies on recruitment, retention, and satisfaction with the intervention are important to determine how and if a full-scale study should be implemented [6]. As such, this paper focuses on the feasibility and acceptability of computer technology in children with ASD who have moderate to low intellectual functioning. A future paper will present data on the efficacy of the intervention from these participants.

\section{Working Memory Impairments in Autism}

ASD is characterized by impairments in communication skills and reciprocal social interactions, and restricted, repetitive, and stereotyped patterns of behavior and interests [7]. In addition, executive function, which is responsible for organizing and regulating behaviors [8-10], has been emphasized as a core dysfunction of ASD [10-13]. One important component of executive functioning is working memory (WM), which is involved in maintaining and manipulating incoming information during planning and executing cognitive tasks [14,15]. WM performance is highly predictive of individual differences in verbal and visuospatial memory span and reasoning [16], general fluid intelligence [17], reading comprehension difficulties [18-21], mathematical difficulties [22-24], and attention deficit hyperactivity disorder (ADHD) symptoms [25,26]. Andersen et al [27] showed that verbal WM ability in children with high-functioning ASD does not demonstrate the typical developmental increases in capacity expected over a 2-year time period [27] found in children with ADHD or typically developing (TD) children. In other studies, children with ASD had lower spatial working memory training (WMT) abilities than TD children [28,29].

Although WM capacity was previously assumed to be a stable trait [30,31], more recent studies have suggested that WM capacity can be increased through targeted training [32-35]. WMT has been shown to be beneficial in many populations, including individuals within ADHD [36-40], Down syndrome $[41,42]$, and fetal alcohol disorders [43]. However, the efficacy of WMT is currently debated. For example, a number of reviews and meta-analyses identified limitations of the research in WMT $[34,44,45]$, such as the lack of methodological consistency between studies [34] and inconsistency in generalization of benefits to other cognitive domains $[36,44,45]$.

Few studies have examined the effects of computerized WMT in children with ASD [3,46]. de Vries and colleagues [3] concluded that WMT is not feasible for children with ASD, based on their sample (8-12 years of age; IQ> 80) as evinced by a high attrition rate $(26 \%)$ and marginal improvement on near-transfer WM and ADHD behavior. However, the findings were limited by the absence of assessment of motivation. Because high motivation and a positive attitude are critical components to successful training $[32,44]$, it is possible that the high attrition rate may have been due to low motivation.

\section{Addressing Gaps in Working Memory Training in Autism}

Another limitation in the existing cognitive training literature is the lack of research on children with ASD and intellectual disability (ID), which is crucial considering that over a third of individuals with ASD also have ID [1]. The aforementioned de Vries study [3] included children with intellectual functioning of greater than 80 . Understanding feasibility of training is valuable for exploring the viability of translating computerized interventions, such as WMT, into clinical practice. To this end, this study assessed a computerized cognitive training program for feasibility, as measured by attrition rate and parent responses, in children with ASD with low to moderate intellectual ability.

\section{Methods}

\section{Participants}

Participants were recruited through the MIND Institute's Subject Tracking System, flyers located at the local clinic, Alta California Regional Center, and advertisements placed in websites and local newspapers (Figure 1). In total, 91 volunteers were assessed for eligibility via a brief series of questions with the parent of the potential participant over the phone. The inclusion criteria included children aged 8-17 years with a diagnosis of ASD, below average intellectual functioning (IQ $\leq 85$ ), and normal to corrected normal vision and hearing. Participants were excluded if there were plans to change current behavioral or pharmacological treatment during the course of the study, if a caregiver reported disruptive behaviors that would interfere with WMT, or if the participant was unable to use a computer or tablet. A total of 37 of the 91 volunteers met the eligibility criteria and underwent an in-person assessment visit. Participants were further excluded at the in-person assessment if observable self-injurious behaviors were present or if their 
IQ scores were greater than 85 . Most of the volunteers $(n=54)$ were excluded because their IQ was greater than 85. A total of 26 study participants (mean age 11.1 [SD 2.4] years) remained after exclusions. This sample included 21 males and 5 females, which reflects the ratio found in the ASD population [1]. The average IQ for the sample was 65 (range: 47-85; SD 14; Table 1).

Intellectual functioning was determined by current or previous testing, if it was available within the past 3 years with the Wechsler Intelligence Scale for Children, Fourth Edition, or
Stanford-Binet Intelligence Scales, Fifth Edition (SB-5). The verbal and nonverbal routing subtests of the SB-5 were administered to estimate the abbreviated IQ for participants without recent testing. The diagnosis of ASD was confirmed by documentation of previous assessments (The Autism Diagnostic Observation Schedule or the Autism Diagnostic Interview-Revised). If no prior diagnosis had been made, ASD diagnosis was confirmed through the Social Communication Questionnaire Lifetime (SCQ), for which the total SCQ score was greater than 15 .

Figure 1. Flow of participant recruitment, participation, and outcomes assessed in feasibility study. WMT: working memory training; ASD: autistic spectrum disorder.

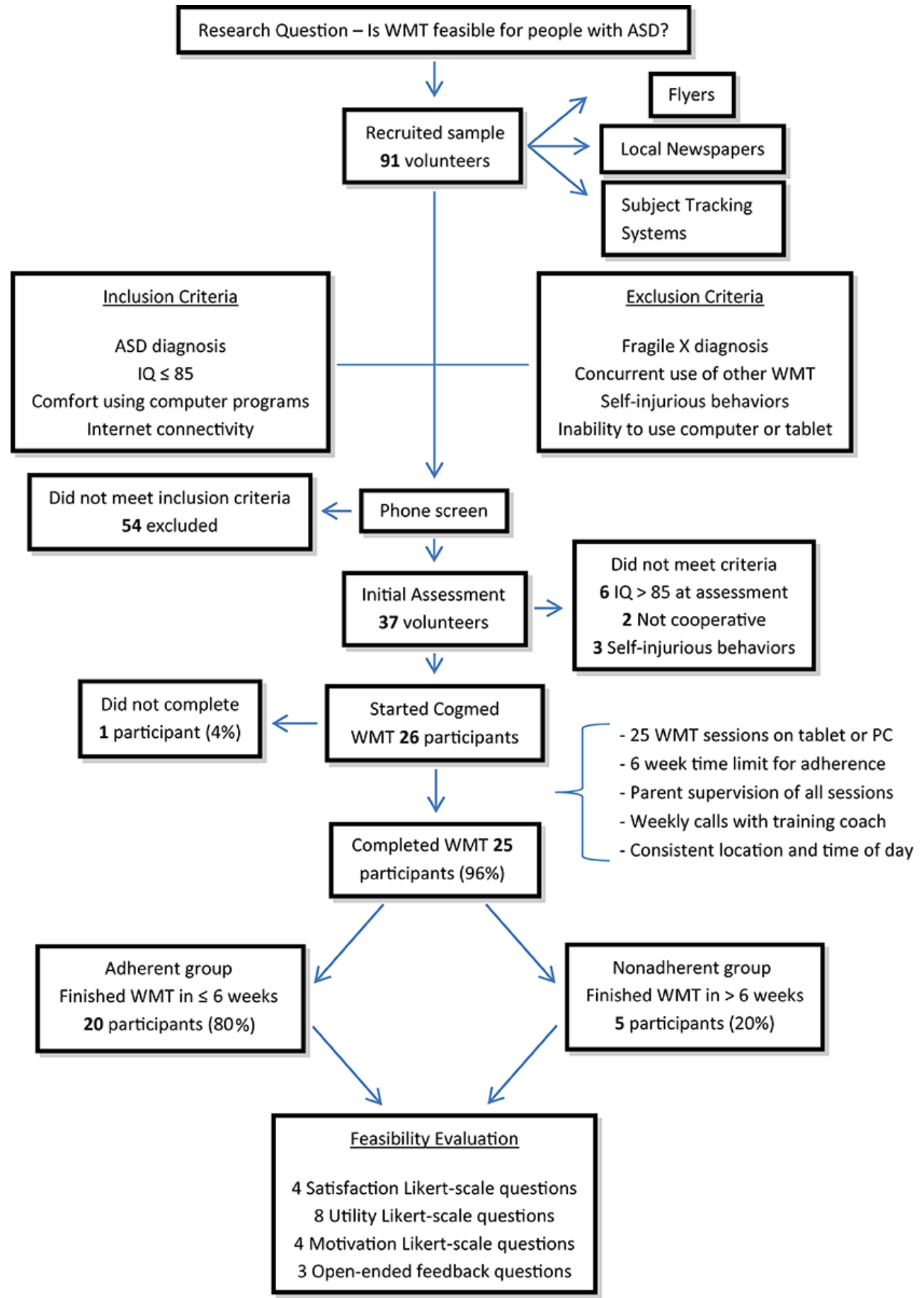


Table 1. Characteristics of participants $(\mathrm{N}=26)$.

\begin{tabular}{|c|c|}
\hline Variable & Summary \\
\hline Age in years, mean $(\mathrm{SD})$ & $11.1(2.4)$ \\
\hline \multicolumn{2}{|l|}{ Gender, n (\%) } \\
\hline Female & $5(19)$ \\
\hline Male & $21(81)$ \\
\hline \multicolumn{2}{|l|}{$\operatorname{Race}^{\mathrm{a}}, \mathbf{n}(\%)$} \\
\hline Black & $3(12)$ \\
\hline White & $14(56)$ \\
\hline Asian & $5(20)$ \\
\hline Other & $3(12)$ \\
\hline Intelligence quotient (IQ), mean (SD) & $65(14)$ \\
\hline \multicolumn{2}{|l|}{ Training device, $n(\%)$} \\
\hline Tablet & $18(69)$ \\
\hline Personal computer (PC) & $8(31)$ \\
\hline \multicolumn{2}{|l|}{ Cogmed version, $\mathbf{n}(\%)$} \\
\hline $\mathrm{JM}^{\mathrm{b}}$ & $15(58)$ \\
\hline $\mathrm{RM}^{\mathrm{c}}$ & $11(42)$ \\
\hline \multicolumn{2}{|l|}{ Current therapies, n (\%) } \\
\hline Occupational therapy & $15(58)$ \\
\hline Physical therapy & $4(15)$ \\
\hline Speech therapy & $21(81)$ \\
\hline Applied behavioral analysis & $11(42)$ \\
\hline \multicolumn{2}{|l|}{ Psychotropic medications, n (\%) } \\
\hline ADHD $^{\mathrm{d}}$ stimulant & $1(4)$ \\
\hline ADHD nonstimulant & $4(15)$ \\
\hline Antidepressant & $3(12)$ \\
\hline Antipsychotic & $6(23)$ \\
\hline Mood stabilizer & $3(12)$ \\
\hline Other medications & $2(8)$ \\
\hline No medications & $15(58)$ \\
\hline \multicolumn{2}{|l|}{ Comorbidities, n (\%) } \\
\hline ADHD & $6(23)$ \\
\hline Mood disorders & $2(8)$ \\
\hline Anxiety disorders & $3(12)$ \\
\hline Seizure disorders & $3(12)$ \\
\hline Other mental disorders & $2(8)$ \\
\hline Other physical disorders & $2(8)$ \\
\hline \multicolumn{2}{|l|}{ Completion and adherence, $\mathrm{n}(\%)$} \\
\hline Completed training $^{\mathrm{e}}$ & $25(96)$ \\
\hline Adherent to protocol ${ }^{\mathrm{f}}$ & $19(73)$ \\
\hline Nonadherent ${ }^{\mathrm{f}}$ & $5(19)$ \\
\hline
\end{tabular}




\begin{tabular}{ll}
\hline Variable & Summary \\
\hline Did not complete training $^{\mathrm{e}}$ & $1(4)$ \\
Combined attrition $^{\mathrm{g}}$ & $6(23)$ \\
\hline
\end{tabular}

${ }^{a}=1$ did not provide an answer.

${ }^{\mathrm{b}} \mathrm{JM}$ : Cogmed for preschool-aged children.

${ }^{\mathrm{c}} \mathrm{RM}$ : Cogmed for school-aged children.

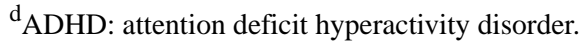

${ }^{\mathrm{e}}$ Required completing 25 sessions as reported by the Cogmed website.

${ }^{\mathrm{f}}$ Required completion of 25 sessions within 6 weeks of start date.

${ }^{\mathrm{g}}$ Not completing training or not adhering to protocol.

\section{Procedure}

All procedures of the study were reviewed and approved by the University of California, Davis Institutional Review Board. After screening for criteria with the parents over the phone, parents provided written informed consent and participants over the age of 11 years provided assent. Tests assessing baseline WM, cognitive abilities, attentional functioning, and autism symptoms were administered at the participants' homes. After participants completed Cogmed training, researchers administered the same test battery to assess training effects. Data on training effects on objects measures of performance and rating scales will be presented in a separate publication. Parents completed posttraining surveys to evaluate feasibility of WMT. Participants were reimbursed US \$20 for their time in the initial assessment and US $\$ 50$ for the follow-up assessment.

\section{Intervention}

All participants were instructed to complete 5 Web-based Cogmed Working Memory Training [47] sessions per week for 5 weeks, for a total of 25 training sessions. There were 2 difficulty levels for participants. Cogmed RM was developed for school-aged TD children, whereas Cogmed JM was for preschool TD children. Those who comprehended 9 of the 11 Cogmed RM games, as determined by researchers during the in-person assessment, were assigned to Cogmed RM ( $n=15)$, with the remainders assigned to Cogmed JM $(n=11)$. The Cogmed JM groups required training for $15 \mathrm{~min}$ per session and the Cogmed RM group required training for 30 min per session. Participants were trained either on the Cogmed tablet app ( $n=18$; use of finger for item responses), with tablets provided as necessary, or on the Cogmed website ( $n=8$; use of $\mathrm{PC}$ with a mouse for item responses). See Table 1 for additional information.

Each training session was conducted at home in a location with limited distractions and parental supervision. As per the Cogmed's protocol, parents were trained as training aides by Cogmed coaches to ensure participants' motivation and focus. Researchers who underwent Cogmed Research Coach Training contacted parents by phone or email at least once a week to address concerns related to training. These coaches had online access to participants' frequency of use and performance on Cogmed tasks to track progress and provide feedback as necessary. Coaches also answered questions regarding software issues and took general feedback about use of the program.

Both Cogmed versions involved visuospatial WM span tasks, and, additionally, the RM version included verbal WM span tasks. Daily Cogmed JM training involved completing 3 of the $7 \mathrm{JM}$ games, whereas the RM training involved the completion of 8 of the 10 RM games, with games rotated in each session to maintain novelty and interest. Both versions were adaptive; the difficulty gradually increased after correct trials and decreased after incorrect trials. Both versions emitted auditory and visual feedback after each trial to indicate success or failure at the task. After the completion of each training session, Cogmed JM users received a virtual fish for their digital aquarium, and Cogmed RM users played a racing game as a reward. For added motivation, parents gave a sticker after each session for a reward chart, and parents and participants decided on daily, weekly, and full training completion rewards.

\section{Measures}

\section{Completion, Adherence, and Combined Attrition}

The completion rate was defined as the percentage of participants who completed 25 sessions of Cogmed WMT. Although it is recommended by Cogmed that training should be completed in 5 weeks, training duration was extended to 6 weeks; it was presumed that participants would require more time to complete the training due to learning and behavioral challenges associated with ASD and ID. Participants were considered "adherent" if they finished 25 sessions within 6 weeks. Participants who took longer than 6 weeks to complete the training were considered "nonadherent" with the training protocol. The combined attrition rate was defined as the percentage of participants who did not complete 25 sessions and who were nonadherent with the training protocol.

\section{Posttraining Survey}

At the end of training, parents completed a questionnaire with 16 Likert-scale and 3 open-ended questions. The questionnaire, adapted from a previous ADHD intervention study [48], aimed to measure subjective opinions regarding the feasibility of the program. Likert-scale questions comprised a 5-point scale (1=strongly disagree, $2=$ disagree, $3=$ neutral, 4=agree, and $5=$ strongly agree) and inquired into participant motivation, parent satisfaction, and perceived utility. We summarized the responses by calculating both mean and SD and the percentage of parents who answered agree/strongly agree (satisfied/strongly satisfied), were neutral, or answered disagree/strongly disagree (dissatisfied/ strongly dissatisfied) for each question. 
Open-ended questions were asked about the pros and cons of the program and possible improvements.

\section{Statistical Analysis}

Data were collected and managed using REDCap electronic data capture tools hosted at University of California, Davis [49]. Feasibility of Cogmed training was assessed both quantitatively and qualitatively. Quantitatively, descriptive statistics were calculated for the completion rate and Likert-scale questions. Qualitatively, deidentified data from open-ended questions were used for content analysis. Initially, phrases taken from the open-ended responses were tagged with low-level codes. These codes were compared and found to fit into 3 broad categories based on previous qualitative literature in computer-based training [50]: technological features, personal experiences, and strategy of use.

\section{Results}

\section{Attrition}

A total of 25 of the 26 participants (96\%) completed all 25 sessions of Cogmed WMT. Of these 26 participants, 20 (77\%) completed the WMT within 6 weeks and were considered adherent to protocol, 5 (19\%) took more than 6 weeks and were considered nonadherent, and 1 participant (4\%) completed only 16 sessions due to unsolved technical problems. The combined attrition rate (counting those who did not complete 25 sessions and did not complete the training within 6 weeks) was $23 \%$. The combined attrition rates were similar across Cogmed JM and RM users. Of the $15 \mathrm{JM}$ participants, 3 were considered nonadherent and 1 did not complete the training (combined attrition rate $=27 \%$ ). All $11 \mathrm{RM}$ participants completed the training, but 3 were considered nonadherent (combined attrition rate $=27 \%$ ).

\section{Posttraining Ratings}

Likert-scale questions queried parents about the 3 domains of satisfaction, perceived utility, and motivation (Table 2). The percentage of parents who agreed or strongly agreed with questions regarding program satisfaction was at least $88 \%$ for each item. Responses to questions about the perceived utility of the training were less consistent. There was mixed evidence about improvements in daily life (child's attention/behavior, peer relations, study skills, homework, self-esteem/attitude, and relationship with the parent), but most parents reported that they observed improvement on performance on the program itself (76\% agree) and felt the WMT approach was appropriate for their child (84\% agree). Regarding motivation, parents generally thought that their children found Cogmed to be enjoyable, with $72 \%$ of respondents agree to this item. However, many parents found it hard to maintain their children's motivation to train on a daily basis. The item asking whether it was easy to keep the child motivated, received a high number of "disagree" responses
(28\%); similarly, only $32 \%$ of parents said that the child's motivation improved by end of training.

The pattern of parent responses was similar for Cogmed JM and RM versions, with a few exceptions. Parents of children assigned the JM version perceived more improvement in motivation and less improvement in homework (Multimedia Appendix 1). It is important to note, however, that only 19 of the 25 parents responded on the item asking about child's homework improvement, as some children were not assigned homework during the summer months of data collection. We also examined (data not shown) the responses of the 5 participants with comorbid ADHD who completed the training, and they were consistent with the pattern observed for those without comorbid ADHD.

\section{Posttraining Feedback}

Content in open-ended responses was analyzed and classified into 3 main areas: (1) technological features, (2) personal experiences, and (3) usage strategies.

\section{Technological Features}

Many of the parents had comments about game design, system functionality, and rewards. Comments about game features included discussion about the graphics and sound. One parent said her child enjoyed the "giggling sounds"; multiple participants liked the voice-over. Moreover, parents liked the reward games that children accessed when all daily tasks were finished. However, participants also reacted negatively to some stimuli, such as the cow sounds or spider graphics. Another area that parents noted as need improvement was game variability. Several parents indicated that motivation could be improved either by varying the games or by giving the child a choice about which game to play each day. Moreover, another common theme among technology-related comments was that there were "glitches" or "bugs" in the Cogmed WMT website.

\section{Personal Experiences}

Several parents indicated that the WMT affected daily functioning in their children. Several described perceived increased abilities in their children associated with using WMT, such as increased focus, memory, and reaction time. However, some parents saw strong negative emotional reactions in their children. Tantrums, low motivation, and frustration were all reported, though pride at completing difficult tasks was also mentioned.

\section{Usage Strategies}

Cogmed has several strategies to help improve adherence and outcomes, as described in the Methods section; these strategies generated many comments. For example, imposing breaks could be difficult or turn into a power struggle. Several comments mentioned appreciation of the coaches. Responses to the daily training length varied, with some parents wishing for shorter sessions and others wanting longer. 
Table 2. Summary of parent posttraining ratings for 25 participants who completed all 25 sessions of Cogmed working memory training.

\begin{tabular}{|c|c|c|c|c|}
\hline \multirow[t]{2}{*}{ Item $^{\mathrm{a}, \mathrm{b}}$} & \multirow[t]{2}{*}{ Mean (SD) } & \multicolumn{3}{|c|}{ Frequency $(\%)^{\mathrm{c}}$} \\
\hline & & Agree $^{\mathrm{d}}$ & Neutral $^{\mathrm{e}}$ & Disagree \\
\hline \multicolumn{5}{|l|}{ Satisfaction } \\
\hline Staff show interest and concern & $4.52(0.59)$ & $24(96)$ & $1(4)$ & $0(0)$ \\
\hline Staff are skilled & $4.56(0.58)$ & $24(96)$ & $1(4)$ & $0(0)$ \\
\hline Treatment is of high quality & $4.24(0.93)$ & $22(88)$ & $2(8)$ & $1(4)$ \\
\hline I would recommend this to others & $4.20(0.58)$ & $23(92)$ & $2(8)$ & $0(0)$ \\
\hline \multicolumn{5}{|l|}{ Perceived utility } \\
\hline Child's attention/behavior improved & $3.56(0.65)$ & $14(56)$ & $10(40)$ & $1(4)$ \\
\hline Child's peer relations improved & $3.32(0.63)$ & $8(32)$ & $16(64)$ & $1(4)$ \\
\hline Child's study skills improved ${ }^{\mathrm{f}}$ & $3.35(0.57)$ & $7(30)$ & $16(70)$ & $0(0)$ \\
\hline Child's homework improved ${ }^{\mathrm{g}}$ & $3.47(0.61)$ & $8(42)$ & $11(58)$ & $0(0)$ \\
\hline Child's self-esteem/attitude improved & $3.32(0.63)$ & $8(32)$ & $16(64)$ & $1(4)$ \\
\hline Relationship with child improved & $3.44(0.58)$ & $10(40)$ & $15(60)$ & $0(0)$ \\
\hline Child made progress in training & $3.88(0.60)$ & $19(76)$ & $6(24)$ & $0(0)$ \\
\hline $\mathrm{WMT}^{\mathrm{h}}$ approach is appropriate & $3.96(0.68)$ & $21(84)$ & $3(12)$ & $1(4)$ \\
\hline \multicolumn{5}{|l|}{ Motivation } \\
\hline Child enjoyed the training & $3.76(0.78)$ & $18(72)$ & $5(20)$ & $2(8)$ \\
\hline Easy to keep child motivated & $3.44(1.08)$ & $14(56)$ & $4(16)$ & $7(28)$ \\
\hline Training is as enjoyable as commercial games & $2.64(0.95)$ & $4(16)$ & $6(24)$ & $15(60)$ \\
\hline Child's motivation improved by end of training & $2.88(1.01)$ & $8(32)$ & $6(24)$ & $11(44)$ \\
\hline
\end{tabular}

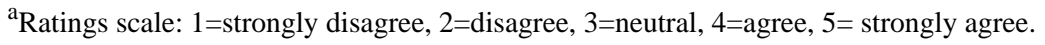

${ }^{\mathrm{b}}$ Item descriptions paraphrase actual questions.

${ }^{\mathrm{c}}$ Percentages may not add to $100 \%$ due to rounding.

${ }^{\mathrm{d}}$ Percent agree indicates percentage of sample responding with 4 or 5 .

${ }^{\mathrm{e}}$ Percent disagree indicates percentage of sample responding with 1 or 2 .

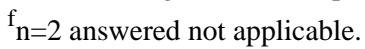

$\mathrm{g}_{\mathrm{n}}=6$ answered not applicable.

${ }^{\mathrm{h}}$ WMT: working memory training.

\section{Discussion}

The purpose of this pilot study was to examine, for the first time, the feasibility of using a cognitive training program in children with ASD with low to moderate intellectual functioning. Although the vast majority of participants (96\%) were able to complete the 25 Cogmed sessions, many of them (24\%) needed more than 6 weeks, which is an extension of the 5-week period recommended by Cogmed. This suggests that although completion may be feasible for children with ASD, Cogmed's suggested training schedule may be too aggressive for this population.

Using a strict time requirement of completion within 6 weeks resulted in an attrition rate similar to the attrition rates found in previous research on children with ASD and no intellectual impairment (26\%) [3] and children with ADHD (23\%) [36]. In these previous studies, it is unclear whether participants were given the option of finishing training beyond 6 weeks; thus, the overall completion rate in this study $(96 \%)$ is not directly comparable. However, WMT studies that incorporated a flexible time requirement with other clinical populations reported similar completion rates. For example, Conklin et al [51] found that $88 \%$ of childhood cancer survivors completed training in 5-9 weeks. Also of note, Mawjee et al [52] found that children with ADHD had a higher Cogmed completion rate $(78 \%)$ and showed more motivation when given shorter training lengths (15 $\mathrm{min} /$ session) than did those who were assigned standard-length training (45 min/session; 44\% completion). In this study, participants assigned the JM version (15 min/session) had similar completion rates as those assigned the RM version (30 $\mathrm{min} / \mathrm{session}$ ), but similar to the findings of Mawjee et al [52], parents of children assigned the JM version perceived more improvement in their child's motivation than those assigned the $\mathrm{RM}$ version.

The sample in this study had significant levels of cognitive delay (mean $I Q=65$ ), and thus may have required more time to 
complete the program due to the presence of learning difficulties. In support of this idea, Bennett et al [41] showed that a sample of children with Down syndrome who had a mean IQ less than 70 took 10-16 weeks to complete Cogmed WMT. Therefore, an appropriate training schedule should be further investigated for successful WMT implementation in populations with developmental delays.

Qualitative measurement of feasibility, as measured by parental survey, revealed overall satisfaction with the WMT. More than $85 \%$ of parents agreed with each satisfaction-related item, which is similar to findings from other studies that evaluated the feasibility of WMT in other populations [51,53]. On the basis of the open-ended responses in this study, it appears that in-home training, flexibility to set a preferred training time, and weekly coaching calls contributed to higher parent satisfaction. Although most parents reported that their children enjoyed the training, only half felt it was easy to keep their children motivated. This may be due to training difficulty, length of training (eg, RM vs JM), or technical problems.

Parents consistently reported agreement with items regarding perceived improvement in child attention and behavior and progress in training, but they reported mainly neutral responses to questions regarding improvement in academic or interpersonal relationship skills. This finding is consistent with the results in the study by Chacko et al [36], which showed Cogmed resulted in improvement in WM but did not transfer to improvement in daily life skills.

This study cannot make any conclusions regarding the effectiveness of WMT in children with ASD and ID. The goal of this study was to determine whether this population can engage in cognitive training in a home setting to permit future evaluation of the effectiveness of training. A future manuscript will present findings on the effectiveness of this training.

On the basis of the findings from the parent surveys, the authors suggest the following solutions for the successful implementation of computerized cognitive training programs for children with ASD. First, the training program should be deployable in such a way as to be nonaversive to children who have sensitivities to visual and auditory stimuli. Due to sensory processing issues, which are often found in children with ASD, some parents reported that their children found certain graphics (eg, spiders) and sounds (eg, "Boo" after an incorrect response) distressing. These stimuli were unavoidable; games could not be skipped and participants were not able to turn off sounds due to program's use of auditory instructions. WMT programs should give users the option to edit graphics and sound settings or to skip games that induce distress.

Second, technical problems should receive high priority. Due to the characteristics associated with ASD, such as inflexibility and difficulty with routine change [7], technical problems may interfere more with adherence and motivation among individuals with ASD than among other populations. Indeed, open-ended responses showed that these problems often triggered frustration. The only participant who did not complete the training program experienced a software crash, which erased the record of his work, causing him to quit permanently. Individuals with ASD may require a training program that is more fault-tolerant and less liable to crash.

Third, the use of behavior management techniques may facilitate adherence with computer training in this population. Certain behavioral problems such as temper tantrums and self-injurious behavior can hinder training. Although the coaches provided guidance on reinforcement techniques, additional behavioral strategies such as stimulus control, extinction, or relaxation training may further decrease the likelihood of disruptive behaviors and increase adherence to protocol.

Fourth, the training should be more intrinsically motivating to maintain the interest of children with ASD and ID. Because this study utilized the adaptive version of Cogmed, in which the difficulty level gradually increased after correct consecutive trials, participants were working at peak level at all times. To keep the interest, it is crucial that the training is also highly motivating. The posttraining surveys indicate that keeping participants motivated to use Cogmed WMT was difficult, and that Cogmed was not as enjoyable as commercially available games. We suggest that both of these issues can be improved with better game design; program developers should create more motivating gameplay or use interesting characters, stories, and goals to maintain participants focus.

\section{Limitations}

Although this study offers novel information on a population that has been understudied in the cognitive training research, the findings must be interpreted in light of limitations. Because there was no control group, self-report biases may have inflated some feasibility responses. Future studies should consider a systematized qualitative design, such as a focus group, to provide more comprehensive information. Moreover, the sample size was relatively small; thus, increasing the number of participants in future research may further enhance the generalizability of the present findings. Future research should also consider including more severe cases of ASD, as some individuals were excluded due to self-injurious behavior or high levels of noncompliance.

The funding for this project was specifically given by the agency to fund feasibility projects to assess if potential interventions would be successful and worth exploring in larger, controlled design for individuals with autism. This paper met those requirements in addition to a sister manuscript, which is under preparation, assessing the effects of the cognitive training on objective measures of performance and rating scales.

\section{Conclusions}

Assessing stakeholder perspectives in intervention research is critical throughout the design and implementation process. The development of computerized cognitive training procedures for children with ASD is likely to increase due to their accessibility. This feasibility study is the first to systematically evaluate the feasibility and parent evaluation of cognitive training among children with ASD and ID. Findings suggest greater feasibility if a flexible training schedule is used in future efficacy studies. In addition, although most parents reported satisfaction with the program assessed in this project, many parents reported software glitches and poor tolerance of graphics and sounds by 
their children. This may be an issue more unique to children with ASD. Thus, parent satisfaction may be improved if users are given greater control over the graphics, sounds, and interactions with specific stimuli. Developers of gaming software for training in ASD would be wise to employ focus groups to gather feedback on how the game stimuli are perceived. Future research will need to assess the efficacy, effectiveness, and generalizability of computerized cognitive interventions for children with ASD.

\section{Acknowledgments}

The authors gratefully acknowledge the families who participated in this research, and research assistants Hilary Sisson, Jaewon Kim, Samantha Schauer, Mellisa Villacarte, and Letitia Pirau for help with data collection, entry, and scoring. The authors thank Cogmed-Pearson for providing the software. Pearson is the owner and copyright holder of the Cogmed Working Memory Training system. Cogmed and Cogmed Working Memory Training are trademarks, in the United States and/or other countries, of Pearson Education, Inc or its affiliate(s). Funding and support for this work were provided by the Faculty of Medicine, Srinakharinwirot University; the Department of Defense (DOD) through a pilot award (AR120332 to Schweitzer); the MIND Institute Intellectual and Developmental Disabilities Research Center (U54 HD079125); the National Center for Advancing Translational Sciences; and National Institutes of Health (NIH) through a grant (UL1 TR001860). The content is solely the responsibility of the authors and does not necessarily represent the official views of the NIH.

\section{Conflicts of Interest}

None declared.

\section{Multimedia Appendix 1}

Summary of parent posttraining ratings by Cogmed version.

[PDF File (Adobe PDF File), 264KB-Multimedia Appendix 1]

\section{References}

1. Developmental Disabilities Monitoring Network Surveillance Year 2010 Principal Investigators, Centers for Disease Control and Prevention (CDC). Prevalence of autism spectrum disorder among children aged 8 years - autism and developmental disabilities monitoring network, 11 sites, United States, 2010. MMWR Surveill Summ 2014 Mar 28;63(2):1-21 [FREE Full text] [Medline: 24670961]

2. National Research Council. Educating Children with Autism. Washington, DC: National Academy Press; $2001: 0309210011$.

3. de Vries M, Prins PJ, Schmand BA, Geurts HM. Working memory and cognitive flexibility-training for children with an autism spectrum disorder: a randomized controlled trial. J Child Psychol Psychiatry 2015 May;56(5):566-576. [doi: 10.1111/jcpp.12324] [Medline: 25256627]

4. Wass SV, Porayska-Pomsta K. The uses of cognitive training technologies in the treatment of autism spectrum disorders. Autism 2014 Nov;18(8):851-871. [doi: 10.1177/1362361313499827] [Medline: 24129912]

5. Kelley M, Edwards K, Starks H, Fullerton SM, James R, Goering S, et al. Values in translation: how asking the right questions can move translational science toward greater health impact. Clin Transl Sci 2012 Dec;5(6):445-451 [FREE Full text] [doi: 10.1111/j.1752-8062.2012.00441.x] [Medline: 23253665]

6. Bowen DJ, Kreuter M, Spring B, Cofta-Woerpel L, Linnan L, Weiner D, et al. How we design feasibility studies. Am J Prev Med 2009 May;36(5):452-457 [FREE Full text] [doi: 10.1016/j.amepre.2009.02.002] [Medline: 19362699]

7. American Psychiatric Association. Diagnostic and Statistical Manual of Mental Disorders (5th ed). Arlington, VA: American Psychiatric Publishing; 2013.

8. Diamond A. Executive functions. Annu Rev Psychol 2013 Jan;64:135-168 [FREE Full text] [doi: 10.1146/annurev-psych-113011-143750] [Medline: 23020641]

9. Hughes C, Russell J, Robbins TW. Evidence for executive dysfunction in autism. Neuropsychologia 1994 Apr;32(4):477-492. [Medline: 8047253]

10. Pennington BF, Ozonoff S. Executive functions and developmental psychopathology. J Child Psychol Psychiatry 1996 Jan;37(1):51-87. [Medline: $\underline{8655658}$ ]

11. Damasio AR, Maurer RG. A neurological model for childhood autism. Arch Neurol 1978 Dec;35(12):777-786. [Medline: 718482]

12. Joseph RM, McGrath LM, Tager-Flusberg H. Executive dysfunction and its relation to language ability in verbal school-age children with autism. Dev Neuropsychol 2005;27(3):361-378 [FREE Full text] [doi: 10.1207/s15326942dn2703 4] [Medline: 15843102]

13. van den Bergh SF, Scheeren AM, Begeer S, Koot HM, Geurts HM. Age related differences of executive functioning problems in everyday life of children and adolescents in the autism spectrum. J Autism Dev Disord 2014 Aug;44(8):1959-1971. [doi: 10.1007/s10803-014-2071-4] [Medline: 24562693] 
14. Baddeley A. The episodic buffer: a new component of working memory? Trends Cogn Sci 2000 Nov 01;4(11):417-423. [Medline: 11058819$]$

15. Barak O, Tsodyks M. Working models of working memory. Curr Opin Neurobiol 2014 Apr;25:20-24. [doi: 10.1016/j.conb.2013.10.008] [Medline: 24709596]

16. Kane MJ, Hambrick DZ, Tuholski SW, Wilhelm O, Payne TW, Engle RW. The generality of working memory capacity: a latent-variable approach to verbal and visuospatial memory span and reasoning. J Exp Psychol Gen 2004 Jun;133(2):189-217. [doi: 10.1037/0096-3445.133.2.189] [Medline: 15149250]

17. Engle RW, Tuholski SW, Laughlin JE, Conway AR. Working memory, short-term memory, and general fluid intelligence: a latent-variable approach. J Exp Psychol Gen 1999 Sep;128(3):309-331. [Medline: 10513398]

18. Cain K. Individual differences in children's memory and reading comprehension: an investigation of semantic and inhibitory deficits. Memory 2006 Jul;14(5):553-569. [doi: 10.1080/09658210600624481] [Medline: 16754241]

19. Gathercole SE, Alloway TP, Willis C, Adams AM. Working memory in children with reading disabilities. J Exp Child Psychol 2006 Mar;93(3):265-281. [doi: 10.1016/j.jecp.2005.08.003] [Medline: 16293261]

20. Gathercole SE, Pickering SJ, Knight C, Stegmann Z. Working memory skills and educational attainment: evidence from national curriculum assessments at 7 and 14 years of age. Appl. Cognit. Psychol 2003 Dec 19;18(1):1-16. [doi: 10.1002/acp.934]

21. Ricketts J. Research review: reading comprehension in developmental disorders of language and communication. J Child Psychol Psychiatry 2011 Nov;52(11):1111-1123. [doi: 10.1111/j.1469-7610.2011.02438.x] [Medline: 21762147]

22. Andersson U, Lyxell B. Working memory deficit in children with mathematical difficulties: a general or specific deficit? J Exp Child Psychol 2007 Mar;96(3):197-228. [doi: 10.1016/j.jecp.2006.10.001] [Medline: 17118398]

23. Dumontheil I, Klingberg T. Brain activity during a visuospatial working memory task predicts arithmetical performance 2 years later. Cereb Cortex 2012 May;22(5):1078-1085. [doi: 10.1093/cercor/bhr175] [Medline: 21768226]

24. Hitch JG, McAuley E. Working memory in children with specific arithmetical learning difficulties. Br J Psychol 1991 Aug;82 (Pt 3):375-386. [Medline: 1954527]

25. Andersen PN, Hovik KT, Skogli EW, Egeland J, Oie M. Symptoms of ADHD in children with high-functioning autism are related to impaired verbal working memory and verbal delayed recall. PLoS One 2013;8(5):e64842 [FREE Full text] [doi: 10.1371/journal.pone.0064842] [Medline: 23717667]

26. Yerys BE, Wallace GL, Sokoloff JL, Shook DA, James JD, Kenworthy L. Attention deficit/hyperactivity disorder symptoms moderate cognition and behavior in children with autism spectrum disorders. Autism Res 2009 Dec;2(6):322-333 [FREE Full text] [doi: 10.1002/aur.103] [Medline: 19998356]

27. Andersen PN, Skogli EW, Hovik KT, Geurts H, Egeland J, Øie M. Working memory arrest in children with high-functioning autism compared to children with attention-deficit/hyperactivity disorder: results from a 2-year longitudinal study. Autism 2015 May;19(4):443-450. [doi: 10.1177/1362361314524844] [Medline: 24604922]

28. Jiang YV, Capistrano CG, Palm BE. Spatial working memory in children with high-functioning autism: intact configural processing but impaired capacity. J Abnorm Psychol 2014 Feb;123(1):248-257. [doi: 10.1037/a0035420] [Medline: $\underline{24661175]}$

29. Steele SD, Minshew NJ, Luna B, Sweeney JA. Spatial working memory deficits in autism. J Autism Dev Disord 2007 Apr;37(4):605-612. [doi: 10.1007/s10803-006-0202-2] [Medline: 16909311]

30. Cowan N. The magical number 4 in short-term memory: a reconsideration of mental storage capacity. Behav Brain Sci 2001 Feb;24(1):87-114; discussion 114-85. [Medline: 11515286]

31. Miller GA. The magical number seven plus or minus two: some limits on our capacity for processing information. Psychol Rev 1956 Mar;63(2):81-97. [Medline: 13310704]

32. Klingberg T. Training and plasticity of working memory. Trends Cogn Sci 2010 Jul;14(7):317-324. [doi: 10.1016/j.tics.2010.05.002] [Medline: 20630350]

33. Klingberg T, Fernell E, Olesen PJ, Johnson M, Gustafsson P, Dahlström K, et al. Computerized training of working memory in children with ADHD--a randomized, controlled trial. J Am Acad Child Adolesc Psychiatry 2005 Feb;44(2):177-186. [doi: 10.1097/00004583-200502000-00010] [Medline: 15689731]

34. Morrison AB, Chein JM. Does working memory training work? The promise and challenges of enhancing cognition by training working memory. Psychon Bull Rev 2011 Feb;18(1):46-60. [doi: 10.3758/s13423-010-0034-0] [Medline: 21327348]

35. Turley-Ames KJ, Whitfield MM. Strategy training and working memory task performance. J Mem Lang 2003 Nov;49(4):446-468. [doi: 10.1016/S0749-596X(03)00095-0]

36. Chacko A, Bedard AC, Marks DJ, Feirsen N, Uderman JZ, Chimiklis A, et al. A randomized clinical trial of Cogmed Working Memory Training in school-age children with ADHD: a replication in a diverse sample using a control condition. J Child Psychol Psychiatry 2014 Mar;55(3):247-255 [FREE Full text] [doi: 10.1111/jcpp.12146] [Medline: 24117656]

37. Cortese S, Ferrin M, Brandeis D, Buitelaar J, Daley D, Dittmann RW, European ADHD Guidelines Group (EAGG). Cognitive training for attention-deficit/hyperactivity disorder: meta-analysis of clinical and neuropsychological outcomes from randomized controlled trials. J Am Acad Child Adolesc Psychiatry 2015 Mar;54(3):164-174 [FREE Full text] [doi: 10.1016/j.jaac.2014.12.010] [Medline: 25721181] 
38. Dovis S, Van der Oord S, Wiers RW, Prins PJ. Improving executive functioning in children with ADHD: training multiple executive functions within the context of a computer game. a randomized double-blind placebo controlled trial. PLoS One 2015;10(4):e0121651 [FREE Full text] [doi: 10.1371/journal.pone.0121651] [Medline: 25844638 ]

39. Green CT, Long DL, Green D, Iosif AM, Dixon JF, Miller MR, et al. Will working memory training generalize to improve off-task behavior in children with attention-deficit/hyperactivity disorder? Neurotherapeutics 2012 Jul;9(3):639-648 [FRE Full text] [doi: 10.1007/s13311-012-0124-y] [Medline: 22752960]

40. Holmes J, Gathercole SE, Place M, Dunning DL, Hilton KA, Elliott JG. Working memory deficits can be overcome: impacts of training and medication on working memory in children with ADHD. Appl Cognit Psychol 2009 Jun 22;24(6):827-836. [doi: 10.1002/acp.1589]

41. Bennett SJ, Holmes J, Buckley S. Computerized memory training leads to sustained improvement in visuospatial short-term memory skills in children with Down syndrome. Am J Intellect Dev Disabil 2013 May;118(3):179-192. [doi:

10.1352/1944-7558-118.3.179] [Medline: 23734613]

42. Comblain A. Working memory in Down syndrome: training the rehearsal strategy. Downs Syndr Res Pract 1994 Oct;2(3):123-126. [doi: 10.3104/reports.42]

43. Loomes C, Rasmussen C, Pei J, Manji S, Andrew G. The effect of rehearsal training on working memory span of children with fetal alcohol spectrum disorder. Res Dev Disabil 2008;29(2):113-124. [doi: 10.1016/j.ridd.2007.01.001] [Medline: 17317098]

44. Melby-Lervåg M, Hulme C. Is working memory training effective? A meta-analytic review. Dev Psychol 2013 Feb;49(2):270-291. [doi: 10.1037/a0028228] [Medline: 22612437]

45. Shipstead Z, Redick TS, Engle RW. Is working memory training effective? Psychol Bull 2012 Jul;138(4):628-654. [doi: 10.1037/a0027473] [Medline: 22409508]

46. Kerns KA, Macoun S, MacSween J, Pei J, Hutchison M. Attention and working memory training: a feasibility study in children with neurodevelopmental disorders. Appl Neuropsychol Child 2017;6(2):120-137. [doi:

10.1080/21622965.2015.1109513] [Medline: 27049769]

47. Cogmed. URL: https://www.cogmed.com/ [accessed 2018-04-24] [WebCite Cache ID 6yviYb2jC]

48. Pfiffner LJ, Kaiser NM, Burner C, Zalecki C, Rooney M, Setty P, et al. From clinic to school: translating a collaborative school-home behavioral intervention for ADHD. School Mental Health 2011 Jun 15;3(3):127-142. [doi: 10.1007/s12310-011-9059-4]

49. Harris PA, Taylor R, Thielke R, Payne J, Gonzalez N, Conde JG. Research electronic data capture (REDCap)--a metadata-driven methodology and workflow process for providing translational research informatics support. J Biomed Inform 2009 Apr;42(2):377-381 [FREE Full text] [doi: 10.1016/j.jbi.2008.08.010] [Medline: 18929686]

50. Stuifbergen A, Becker H, Morgan S, Morrison J, Perez F. Home-based computer-assisted cognitive training: feasibility and perceptions of people with multiple sclerosis. Int J MS Care 2011 Jan 01;13(4):189-198 [FREE Full text] [doi: 10.7224/1537-2073-13.4.189] [Medline: 22740777]

51. Conklin HM, Ogg RJ, Ashford JM, Scoggins MA, Zou P, Clark KN, et al. Computerized cognitive training for amelioration of cognitive late effects among childhood cancer survivors: a randomized controlled trial. J Clin Oncol 2015 Nov 20;33(33):3894-3902 [FREE Full text] [doi: 10.1200/JCO.2015.61.6672] [Medline: 26460306]

52. Mawjee K, Woltering S, Lai N, Gotlieb H, Kronitz R, Tannock R. Working memory training in ADHD: controlling for engagement, motivation, and expectancy of improvement (pilot study). J Atten Disord 2017 Sep;21(11):956-968. [doi: 10.1177/1087054714557356] [Medline: 25501356]

53. Kronenberger WG, Pisoni DB, Henning SC, Colson BG, Hazzard LM. Working memory training for children with cochlear implants: a pilot study. J Speech Lang Hear Res 2011 Aug;54(4):1182-1196 [FREE Full text] [doi: 10.1044/1092-4388(2010/10-0119)] [Medline: 21173394]

\author{
Abbreviations \\ ADHD: attention deficit hyperactivity disorder \\ ASD: autistic spectrum disorder \\ ID: intellectual disability \\ JM: Cogmed for preschool-aged children \\ RM: Cogmed for school-aged children \\ SB-5: Stanford-Binet Intelligence Scales, Fifth Edition \\ SCQ: Social Communication Questionnaire \\ TD: typical developing \\ WM: working memory \\ WMT: working memory training
}


Edited by J Torous; submitted 03.12.17; peer-reviewed by S Bennett, $N$ Khalili-Mahani, M Hochhauser; comments to author 01.02.18; revised version received 01.03.18; accepted 03.03.18; published 25.05.18

Please cite as:

Benyakorn S, Calub CA, Riley SJ, Schneider A, Iosif AM, Solomon M, Hessl D, Schweitzer JB

Computerized Cognitive Training in Children With Autism and Intellectual Disabilities: Feasibility and Satisfaction Study

JMIR Ment Health 2018;5(2):e40

URL: http://mental.jmir.org/2018/2/e40/

doi: 10.2196/mental.9564

PMID: 29802090

CSongpoom Benyakorn, Catrina A Calub, Steven J Riley, Andrea Schneider, Ana-Maria Iosif, Marjorie Solomon, David Hessl, Julie B Schweitzer. Originally published in JMIR Mental Health (http://mental.jmir.org), 25.05.2018. This is an open-access article distributed under the terms of the Creative Commons Attribution License (https://creativecommons.org/licenses/by/4.0/), which permits unrestricted use, distribution, and reproduction in any medium, provided the original work, first published in JMIR Mental Health, is properly cited. The complete bibliographic information, a link to the original publication on http://mental.jmir.org/, as well as this copyright and license information must be included. 